\title{
Contour Fitting of Fused Filaments Cross-Section Images by Lemniscates of Booth: Application to Viscous Sintering Kinetics Modeling
}

\author{
Laurent Chaunier ${ }^{1, *}$, Anne-Laure Réguerre ${ }^{1}$ and Eric Leroy ${ }^{2}$ \\ 1 INRAE, UR BIA, 44316 Nantes, France; anne-laure.reguerre@inrae.fr \\ 2 Université de Nantes, Oniris, CNRS, GEPEA, UMR 6144, 44600 Saint Nazaire, France; \\ eric.leroy@univ-nantes.fr \\ * Correspondence: laurent.chaunier@inrae.fr
}

Citation: Chaunier, L.; Réguerre, A.-L.; Leroy, E. Contour Fitting of Fused Filaments Cross-Section Images by Lemniscates of Booth: Application to Viscous Sintering Kinetics Modeling. Polymers 2021, 13, 3965. https://doi.org/10.3390/ polym13223965

Academic Editor: Jason Bara

Received: 15 October 2021

Accepted: 12 November 2021

Published: 16 November 2021

Publisher's Note: MDPI stays neutral with regard to jurisdictional claims in published maps and institutional affiliations.

Copyright: (c) 2021 by the authors. Licensee MDPI, Basel, Switzerland. This article is an open access article distributed under the terms and conditions of the Creative Commons Attribution (CC BY) license (https:// creativecommons.org/licenses/by/ $4.0 /)$.

\begin{abstract}
A method for image analysis was implemented to determine the edge pixels of two biopolymer-based thermoplastic filaments during their hot melt isothermal sintering at $120{ }^{\circ} \mathrm{C}$. Successive inverted ellipses are adjusted to the contour of the sintered filaments and lead to the identification of the parameters of the corresponding lemniscates of Booth. The different steps of the morphological image analysis are detailed, from 8-bit coded acquired images ( 1 frame/s), to the final fitting of the optimized mathematical functions describing the evolution of the filaments envelope. The complete sequence is composed of an initial pure viscous sintering step during the first minute, followed by viscoelastic swelling combined with melt spreading for a longer time, and then the stabilization of the sintered filaments shape for over $2 \mathrm{~min}$ at high temperatures. Using a master curve obtained from Hopper's abacus, the characteristic viscous sintering time is assessed at $t_{v s}=78 \mathrm{~s}$, confirming the one previously found based on the measurement of the bonding neck length alone. Then, the full description of the evolution of the thermoplastic filaments envelope is assessable by image analysis during sintering trials as a result of its digital modeling as successive lemniscates of Booth, reflecting geometry changes in the molten state.
\end{abstract}

Keywords: biopolymer; fusion-bonding; morphological image processing; parametric equations; viscous sintering

\section{Introduction}

This study deals with the modeling of the viscous sintering of thermoplastic filaments based on a 3D printable biopolymer processed in the molten state. In fused filament deposition-based additive manufacturing processes, the local cross-section between successively deposed filament layers may be considered as a representative element for the description of the mesostructure, affecting the porosity and mechanical properties of the printed parts [1-3]. However, there is currently a lack of equations for the description of such cross-section geometry, and lemniscate curves may be good candidates. Indeed, such algebraic curves have been successfully used by Hopper for the modeling of ceramic sintering kinetics in the case of cylindrical filaments maintaining a constant area when described as a side view [4]. The author gave an exact analytic solution of the plane-flow case where capillarity drives the evolution of the shape of the two cylinders' profile as a function of the sintering time. Starting from this assumption, we propose using the viscous sintering of thermoplastic filaments as a model case to demonstrate the ability of the lemniscates of Booth to fit the contour of the local cross-section and to drastically enhance previous approaches only based on bonding neck length.

The characterization and modeling of viscous sintering kinetics are experiencing a renewed interest due to the development of additive manufacturing technologies based on thermoplastic materials in the form of filaments or powders [5-7]. For this purpose, image 
acquisition systems are set up to follow the coalescence of such divided solid materials in instrumented ovens, or by thermo-microscopy [8-11]. This approach relies on evaluating the length of the bonding neck, $x(\mathrm{~m})$, between two materials as round parts of diameter $d[\mathrm{~m}]$, observed from the side in the case of filaments, or from above in the case of powder particles, and determining their bonding angle, $\theta(\mathrm{rad})$ (Figure 1a):

$$
\theta=\sin ^{-1}\left(\frac{x}{d}\right),
$$

(a)

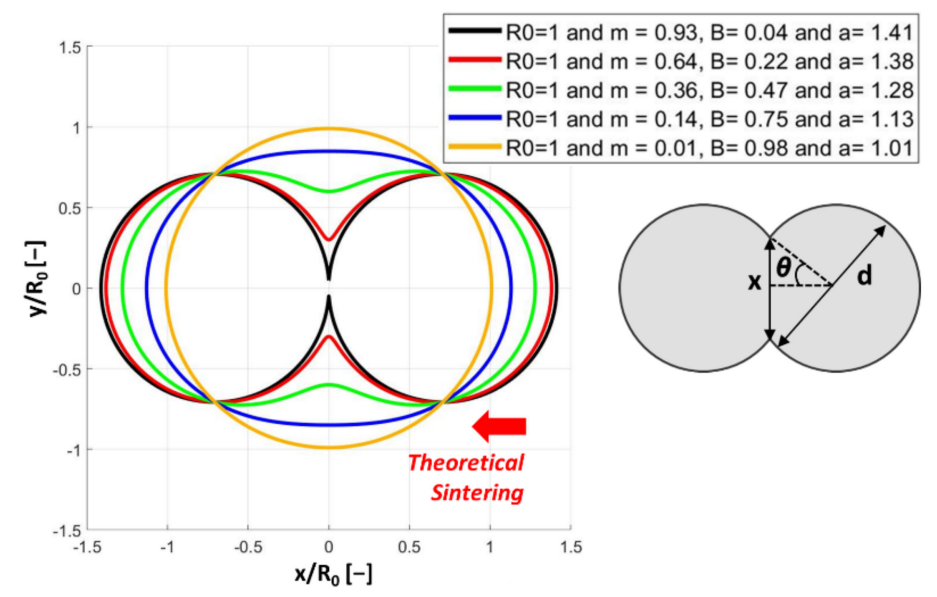

(b)

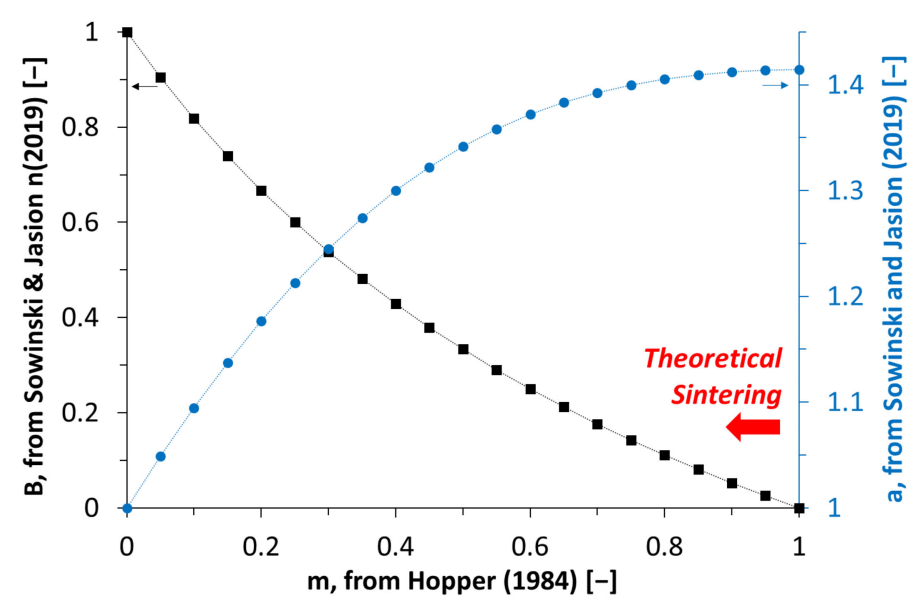

Figure 1. Schematic representation of the theoretical evolution of the contour of two round thermoplastic parts during their viscous sintering as successive inverted ellipses: Lemniscates of Booth presenting a constant area and plotted in a Cartesian coordinate system, with parameter $\mathrm{m}=0.93,0.64$, 0.36, 0.14 and 0.01 (Equation (4), with $\mathrm{R}_{0}=1$ ), the corresponding parameters in a polar coordinate plane: $\mathrm{B}=0.04(\mathrm{a}=1.41), 0.22(\mathrm{a}=1.38), 0.47(\mathrm{a}=1.28), 0.75(\mathrm{a}=1.13), 0.98(\mathrm{~m}=1.01)$-Equation (5)and illustration of the length of the bonding neck, $x$, between the two parts of diameter, $d$, with bonding angle $\theta(\mathbf{a})$. Theoretical evolution of parameters $\mathrm{B}$ and a -Equation (5)-, with $\mathrm{m}$ (in the case of $\mathrm{R}_{0}=1$; from Hopper (1984) [4]) (b). 
The growth rate of the bonding angle can be experimentally assessed during viscous sintering trials. It is then possible to determine the characteristic viscous sintering time, $t_{v s}[\mathrm{~s}]$, by using the tabulated values from the pioneering work of Hopper $[4,12]$ :

$$
\left(\frac{d \theta}{d\left(\frac{t}{t_{v s}}\right)}\right)_{\text {Hopper }}=t_{v s} \times\left(\frac{d \theta}{d t}\right)_{\text {Experimental }},
$$

The term $t_{v s}(\mathrm{~s})$ is linked to the material properties and is defined as follows:

$$
t_{v s}=\left(\frac{R_{i} \times \mu}{\Gamma}\right)
$$

where $R_{i}$ is the initial radius $(\mathrm{m})$ of the thermoplastic material submitted to sintering trials; $\mu$ is the viscosity of the molten material $(\mathrm{Pa} \cdot \mathrm{s})$; and $\Gamma$ is its surface tension $(\mathrm{N} / \mathrm{m})$.

The determination of $t_{v s}$ makes it possible to define the processing window of the considered material, especially to target its additive manufacturing in the molten state. It was recently applied to the deposition of molten filaments based on a thermoplastic biopolymer, zein, plasticized by $20 \%$ glycerol leading to edible and resorbable parts, not accessible with synthetic polymers [12]. $t_{v s}$ was found to drastically decrease, from values above $3500 \mathrm{~s}$ when reaching the molten state at $80^{\circ} \mathrm{C}$, to $t_{v s}<100 \mathrm{~s}$ at temperatures above $120^{\circ} \mathrm{C}$ (i.e., $\mathrm{t}_{\mathrm{vs} \_z e i n} 20 \%$ glycerol_ $120^{\circ} \mathrm{C}=80 \mathrm{~s}$ ). This order of magnitude is similar to the one of a standard amorphous polymer, acrylonitrile butadiene styrene (ABS), used in additive manufacturing at $240{ }^{\circ} \mathrm{C}$, its own printing temperature [12,13]. In this case, the viscous sintering time, $t_{v s}$, was determined by the application of charts established on the evolution of the bonding neck length between two thermoplastic round parts, i.e., two extruded cylindrical filaments observed from the side $\left(\mathrm{R}_{\mathrm{i}},[\mathrm{m}]\right)$ and the relationship to the parametric equations of typical lemniscates curves. These curves represent a series of successive inverted ellipses that evolve during sintering. They correspond to the gradual change in the contour profile of molten round parts [4], as later demonstrated in the literature [14,15]. $t_{v s}$ was found to be on the same order of magnitude as the one determined by the modified Frenkel model, taking the variations in particle diameter during the coalescence step into account $[8,16,17]$. However, to take the entire geometry of the observed system into account, beyond the simple evaluation of the length of the melting front between two thermoplastic filaments brought to high temperatures, this approach must be supplemented by the analysis of their contour as a whole. Furthermore, the geometry characterization that is conventionally used is limited to the initial phase of the sintering sequence because of the apparent swelling of the filaments observed from the side, by an edge effect due to the viscoelastic swelling of melts [18] and their spreading due to gravity over a longer time. To improve this approach, we can link the coordinates of all the points of the edge of the filaments to a theoretical lemniscate of Booth adjusted to the closest of these points. It is expressed in Cartesian coordinates, according to Hopper's approach presented in a vertical layout in his work [4], and adapted in the present work for horizontal juxtaposed filaments:

$$
\left\{\begin{array}{l}
x(\alpha)=\mathrm{R}_{0} \times\left[\left(1-\mathrm{m}^{2}\right) \times\left(1+\mathrm{m}^{2}\right)^{-\frac{1}{2}} \times\left(1+2 \mathrm{~m} \times \cos 2 \alpha+\mathrm{m}^{2}\right)^{-1}\right] \times(1+\mathrm{m}) \times \sin \alpha \\
y(\alpha)=\mathrm{R}_{0} \times\left[\left(1-\mathrm{m}^{2}\right) \times\left(1+\mathrm{m}^{2}\right)^{-\frac{1}{2}} \times\left(1+2 \mathrm{~m} \times \cos 2 \alpha+\mathrm{m}^{2}\right)^{-1}\right] \times(1-\mathrm{m}) \times \cos \alpha
\end{array},\right.
$$

where $\alpha \in[0,2 \pi) ; R_{0}$ is the final radius of the inverted ellipse resulting from the coalescence of the two initial circles of radius $R_{i}$ (with $R_{0}=\sqrt{ } 2 \times R_{i}$, set as constant in order to maintain a constant total section area); and parameter $\mathrm{m} \in[0,1)$.

Such geometry is also described in a polar coordinate system to give more insight into its properties, as recently proposed by Sowinski and Jasion [19]:

$$
r_{p}(\varphi)=a \times \sqrt{\cos ^{2} \varphi+B^{2} \times \sin ^{2} \varphi},
$$

where the parameter a is the size coefficient, and B is the shape parameter (-). $\varphi$ [rad] is defined as $\alpha-\pi / 2$ from Hopper's work in a horizontal layout, with the bonding neck 
placed at $x=0$, and where all of the inverted ellipses have a common point of interception at $2^{-1 / 2} \times R_{0}( \pm 1, \pm 1)$ (Figure 1a). $B \in[0,1]$, corresponding at the edge of this range to Booth lemniscates describing a circle in the case where $\mathrm{B}=1$, or to two connected circles with a tangent point centered on the origin point $(0,0)$ for $\mathrm{B} \rightarrow 0$, or $\mathrm{m} \rightarrow 1$, in the case where $\mathrm{m}=0$ in Hopper's equation (Equation (4); Figure $1 \mathrm{~b}$ ).

The objective of the present study was to set up and implement a novel method for the monitoring of the viscous sintering of cylindrical filaments used in additive manufacturing. It is based on advanced image analysis and modeling of the evolving contour of the filaments' cross-section during the sintering process. The modeling involves a set of constitutive parametric equations of the successive inverse ellipses that match the contour of the filaments.

Compared to previous studies considering the sole measurement and modeling of the evolution of the length of the bonding neck between the filaments $[8,10,12]$, this new approach not only allows estimating the characteristic time for viscous sintering, but also gives an efficient simulation of the shape of the filaments cross-section. Therefore, it could be used to model the local two-layer geometry in printed parts.

For this purpose, this paper describes: (i) the successive steps that allow the determination of the coordinates of the contour edge points of two juxtaposed backlighted filaments placed in an instrumented oven to monitor their hot melt sintering; (ii) the adjustment of the lemniscate corresponding to their contour on each acquired image using the Sowinski and Jasion equation (Equation (4)) in polar coordinates; and (iii) the fine checking of the characteristic viscous sintering time, $t_{v s}$, of the thermoplastic biopolymer, the plasticized zein in this case, used as a model at typical efficient sintering conditions (i.e., $\mathrm{T}=120^{\circ} \mathrm{C}$ ).

\section{Materials and Methods}

\subsection{Plasticized Biopolymer and Filament Extrusion}

The commercial zein powder (Ref. Z3625) and glycerol, used as a plasticizer, were purchased from Sigma-Aldrich (Saint-Quentin-Fallavier, France). They were mixed in a household kneader to obtain the formulation based on zein with $20 \mathrm{w} \%$ glycerol added. This powder blend was extruded with a twin-screw microcompounder at $130{ }^{\circ} \mathrm{C}$ (Haake Minilab, Thermo Scientific GmbH; Karlsruhe, Germany), as previously described [12]. The residence time at $130^{\circ} \mathrm{C}$ was typically about $60 \mathrm{~s}$ before the extrusion of the plasticized zein through a cylindrical die ( $\varnothing_{\text {filament }} \approx 2 \mathrm{~mm}$ ). Differential scanning calorimetry (DSC) experiments showed that the material presents a glass transition with an onset temperature $\mathrm{T}_{\mathrm{g}}=42^{\circ} \mathrm{C}$. This is associated to a main mechanical relaxation occurring at about $45-48{ }^{\circ} \mathrm{C}$, as assessed by dynamic mechanical analysis (DMA) at the peak of the loss modulus E". Above $75-80{ }^{\circ} \mathrm{C}$, the measurement of the moduli is no longer possible in DMA because of material flow. The filaments were cut as cylinders with a constant length, $\mathrm{L}_{\text {filament }}=5 \mathrm{~mm}$, for subsequent fusion-bonding characterization.

\subsection{Sintering Trials}

An instrumented oven was specifically designed for the sintering of two horizontally adjacent extruded filaments, as detailed in previous work, in perfect alignment with the imaging axis. The front of the cavity is a transparent glass plate, while the back is equipped with three LEDs for the backlighting of the two thermoplastic filaments. A CMOS camera allows the acquisition of contrasted images coded on 8 bits, i.e., 256 grey levels, of the cylinder's cross-section ( 1 frame $\cdot \mathrm{s}^{-1}$ ) with an average resolution about $200 \mathrm{pixel} / \mathrm{mm}$.

To monitor the temperature of the filaments $\left(\mathrm{T}_{\text {filament }},\left[{ }^{\circ} \mathrm{C}\right]\right)$, a thin thermocouple (type $\mathrm{K}, \varnothing_{\text {thermocouple }} \approx 0.4 \mathrm{~mm}$ ) was placed inside one of them, previously drilled to its half-length to insert the thermocouple tip. Each experimental sintering trial was carried out at $\mathrm{T}_{\text {set }}=120^{\circ} \mathrm{C}$ in order to create an efficient sintering effect while not expanding the material (i.e., bubbles appearing above $135^{\circ} \mathrm{C}$ to $140{ }^{\circ} \mathrm{C}$, as described in previous work). A second thermocouple (type $\mathrm{K}$ ) was located in the medium part of the oven to monitor the environmental temperature $\left(\mathrm{T}_{\mathrm{oven}},\left[{ }^{\circ} \mathrm{C}\right]\right)$. 


\subsection{Morphological Image Analysis}

Image analysis is applied on the 8-bit coded images (i.e., 256 grey levels) acquired during the sintering of the two juxtaposed filaments placed at $120^{\circ} \mathrm{C}$ (Figure 2a):

- $\quad$ Step 1: The image of the region of interest (ROI) is cropped in order to focus on the two initial filaments and to follow their progressive sintering, while maintaining the initial coding of the image format as 256 grey levels;

- Step 2: A segmentation is carried out by image thresholding in order to obtain binary images with one white object corresponding to the two filaments (i.e., with pixels $=1$ ) on a black background corresponding to the backlighting (i.e., pixels $=0$ ). The constant lighting conditions allow using a single threshold visually determined to segment the whole images sequence with ImageJ software (free software; National Institutes of Health, Bethesda, MD, USA). Based on each binary image, the morphological image analysis (MIA) is applied to determine each point of the contour of the sintered filaments and to fit the adjusted lemniscate to their contour, according to the following steps:

- Step 3: The identification of the contour pixels by an erosion step on the digital mask corresponding to the filament shape with a $3 \times 3$ pixels $^{2}$ structuring element, excluding the oven base;

- Step 4: The determination of the centroid point, i.e., the equivalent of the center of gravity of the white object of the segmented image;

- Step 5: The computation of the maximum horizontal length, $L_{\max }$ [pixel], from one side to the opposite side of the white object on the binary image;

- Step 6: The fitting of a lemniscate of Booth, adapted to the contour of the filaments on each acquired image, implemented on Matlab ${ }^{\circledR}$ software (The MathWorks Inc., Natick, MA, USA). To carry out this last step, a conversion from the initial image coordinate system to a polar centered one is required (Figures $2 b$ and $3 a$ ). Each image is vertically centered on the ordinate at which the horizontal length of the white object is maximum (i.e., where $\mathrm{L}_{\max }$ is determined) and, horizontally, at the abscissa of the centroid, which is equivalent to a central point for the two coalescing filaments.

From the $(x, y)$ centered coordinates of each filaments edge pixel (e.g., point $M$ in the scheme in Figure 2b), the conversion to a polar coordinate system is possible as follows:

$$
\left\{\begin{array}{c}
x=r \times \cos \varphi \\
y=r \times \sin \varphi \\
r=\sqrt{x^{2}+y^{2}}
\end{array},\right.
$$

It is then possible to estimate the parameters of the Lemniscate of Booth equation fitting the contour of the filaments. The least square method of the Matlab ${ }^{\circledR}$ solver was used to optimize the value of the shape parameter $B$ of the polar equation $r_{p}(\varphi)$ of the lemniscate, as proposed by Sowinski and Jasion (Equation (5)), while the evolution of the white object's size during sintering trials is taken into consideration by the size coefficient, $\mathrm{a}=\mathrm{L}_{\max } / 2$. To do so, the parameter $\mathrm{B}$ is determined by minimizing the distance between the contour's pixels and the fitted lemniscate using the fminsearch function of Matlab ${ }^{\circledR}$ software (please see the script detailed in the Electronic Supplementary Materials section, as ESM_1). Then, the two parameters of the fitted lemniscate, a and B, are assessed and the mathematical function of the fitted lemniscate can be plotted. Its constitutive pixels, as well as the ones of the filaments' contour, can be superimposed on each corresponding acquired image, after conversion of their coordinates into the image coordinate system and plotting. This leads finally to each analyzed image (Figures $2 a$ and $3 b$ ). 


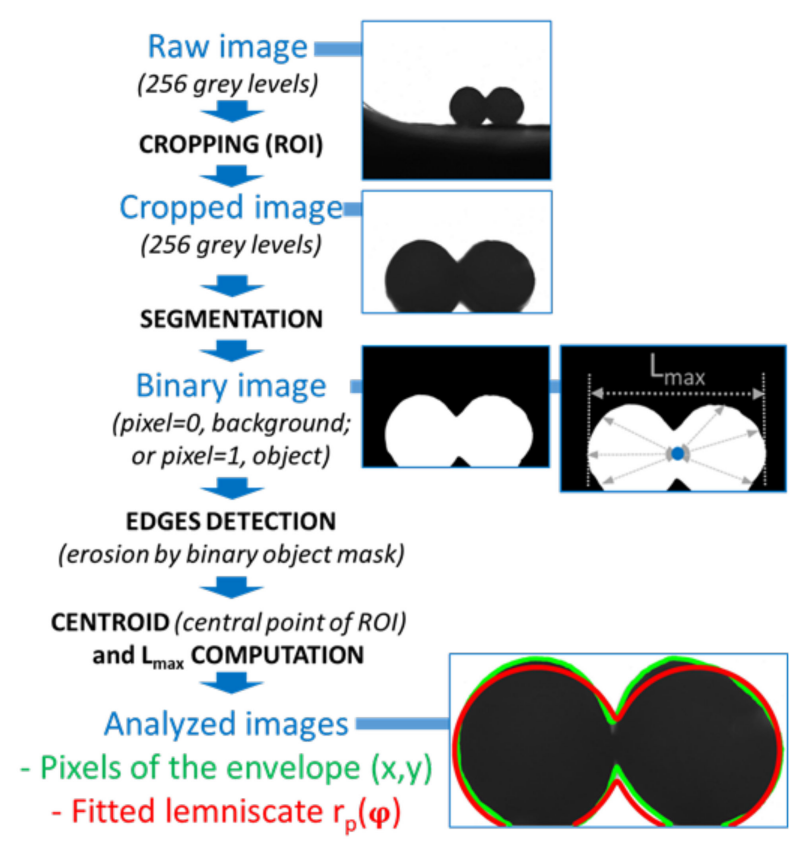

(a)
Image coordinate system

$(0,0)$

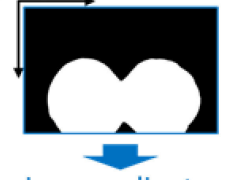

Cartesian coordinate system

$(0,0)$

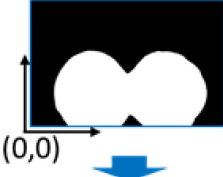

Centered and Polar coordinate systems

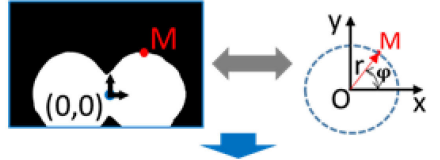

Lemniscate in Polar coordinate system

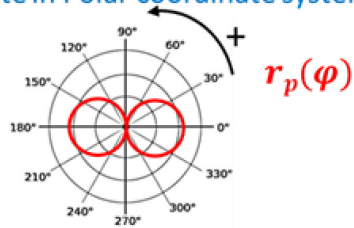

(b)

Figure 2. Image morphological analysis to assess the coalescing filaments contour on each acquired image (-) and its fitted lemniscate of Booth (-) (a). Schematic representation of the successive changes from the segmented -binary-image in a standard image coordinate system to a Cartesian centered one and, finally, to a polar coordinate system (with an example of coordinate conversion taken for one point M), as used in the approach of Sowinski and Jasion [19] (b).

(a)

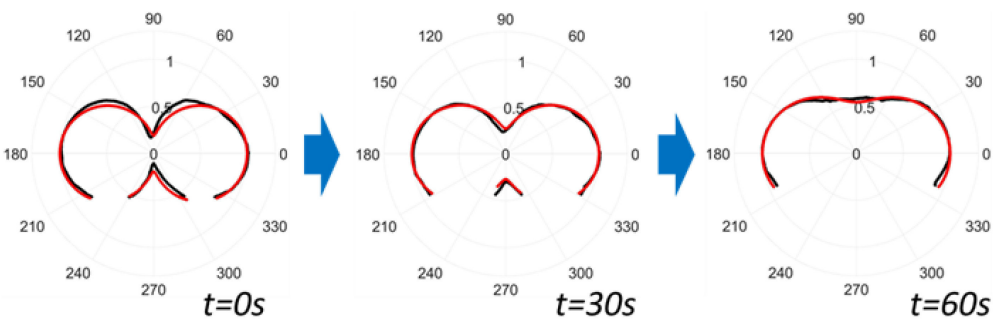

(b)

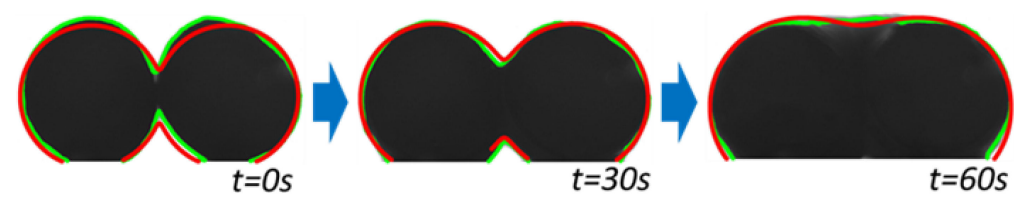

Figure 3. Plot of the successive envelopes of the filaments detected as edge pixels by image analysis $(-)$ and the corresponding fitted lemniscates by Matlab ${ }^{\circledR}$ solver (least squares method) (-) in a polar coordinate system, as in the approach of Sowinski and Jasion [19] (a). Images acquired during the sintering of two thermoplastic filaments at $120^{\circ} \mathrm{C}$ (8-bit coded, i.e., 256 grey levels) and superimposition of their edge $(-)$ and the successive fitted lemniscates of Booth $(-)$ (b) (for corresponding video files, please see the Electronic Supplementary Materials: ESM_2 and ESM_3, respectively).

\section{Results}

\subsection{Successive Lemniscates of Booth to Fit Sintering Sequences}

The use of the least square method to optimize the equation of lemniscates of Booth in a polar coordinate system makes it possible to identify the shape parameter, B, of the 
equation $\mathrm{r}_{\mathrm{p}}(\varphi)$ of Sowinski and Jasion (Equation (5)). Curves correctly match the contour of the filaments during their isothermal sintering, even if changes in shape modify their aspect: First, during their initial pure viscous sintering step, up to 50 to $60 \mathrm{~s}$ at $120^{\circ} \mathrm{C}$, and then when submitted to viscoelastic swelling and spreading for a longer time (Figure 3a; please see the animation in the Electronic Supplementary Materials section, as ESM_2).

When superimposed on the acquired images in their associated coordinate system, the lemniscates of Booth correspond to the edge pixels of the thermoplastic filaments during the complete sequence (Figure 3b; please see the animation in the Electronic Supplementary Materials section, as ESM_3).

\subsection{Determination of the Parameters of the Lemniscate Equations}

Based on the determination of the shape parameter, B, which increases from 0.19 to 0.7 during a 2-min sintering sequence at $120^{\circ} \mathrm{C}$ (Figure $4 \mathrm{a}$; Data available as an accessible dataset cited as reference [20]), it is possible to assess the parameter $\mathrm{m}$ of the equation from Hopper's work (Equation (4)), as follows:

$$
B=\frac{1-m}{1+m},
$$

The monitoring of the coalescence follows the evolution of the shape of the filaments. The values of $\mathrm{m}$ decrease from 0.68 to 0.57 (after $30 \mathrm{~s}$ ), 0.29 (after $1 \mathrm{~min}$ ) and 0.18 (after $2 \mathrm{~min}$ ). At the end of the sintering sequence presented here, the increase in the size coefficient is $112 \%$ of the initial $\mathrm{L}_{\max }$ determined on the first image (namely " $\mathrm{L}_{\text {max }}$ initial" in Figure 4) because of the viscoelastic swelling of the melt and its gravity-driven spreading.

This ratio is stable after about $90 \mathrm{~s}$, as is the shape parameter, typical of the disappearing concavity between the two thermoplastic filaments during their hot melt sintering (Figure $4 \mathrm{~b}$ ). It was checked at the end of each trial carried out at high temperature that the molten sintered parts were not bonded to the metallic surface of the oven when removed.

As detailed in the experimental section, the oven is regulated at $\mathrm{T}_{\text {set }}=120{ }^{\circ} \mathrm{C}$ for these sintering trials. It typically takes $20 \mathrm{~s}$ for the environmental temperature, $\mathrm{T}_{\text {oven }}$ to return to the regulated $T_{\text {set }}$ value once the sintering sequence begins, after closing the glass window of the oven (Figure 4c). It takes more time for the filament core to reach the surrounding temperature, but since the sintering primarily takes place at the filament outline, it is assumed that the peripheral material reaches a steady state after about $20 \mathrm{~s}$, when reaching the molten state [12]. This corresponds to the starting point of the increase of the B parameter from Sowinski and Jasion (Figure $4 b$ ), or the symmetrical decrease of the deduced $m$ parameter from Hopper's work (Figure 4c). 
(a)

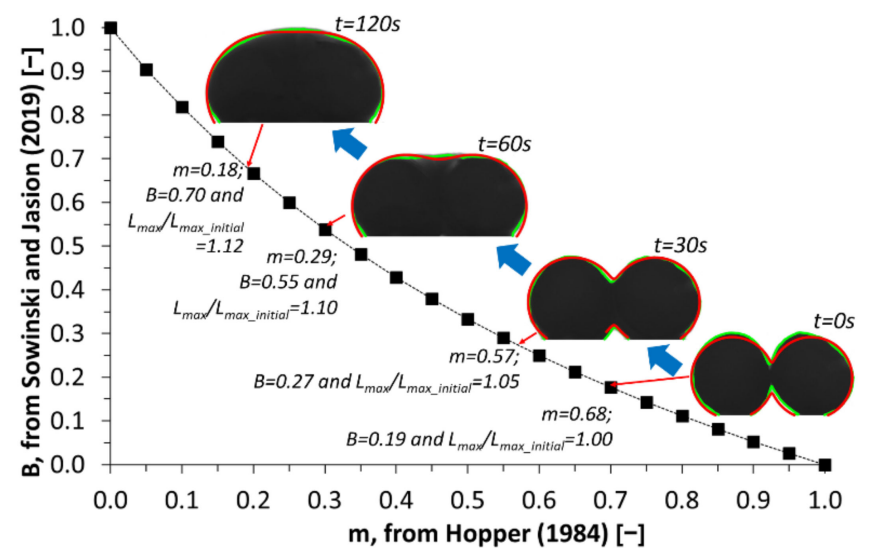

(b)

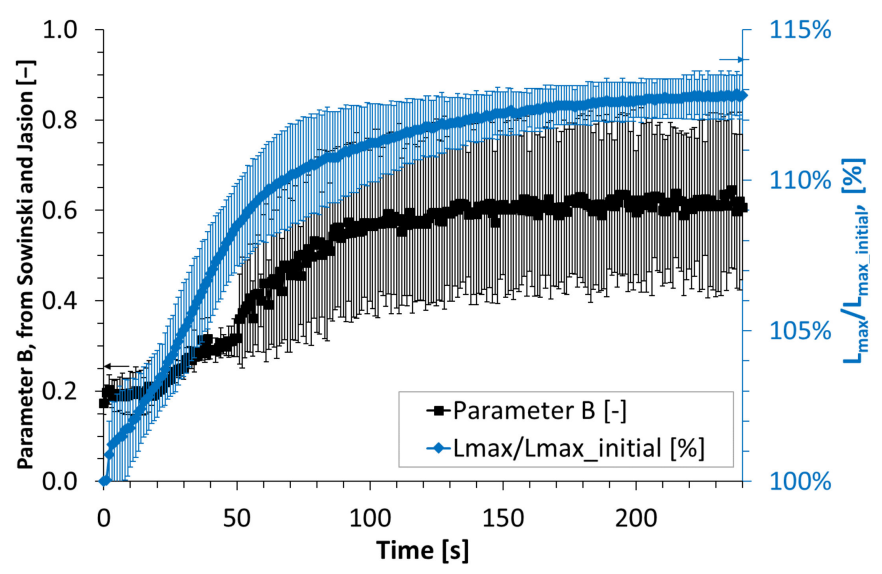

(c)

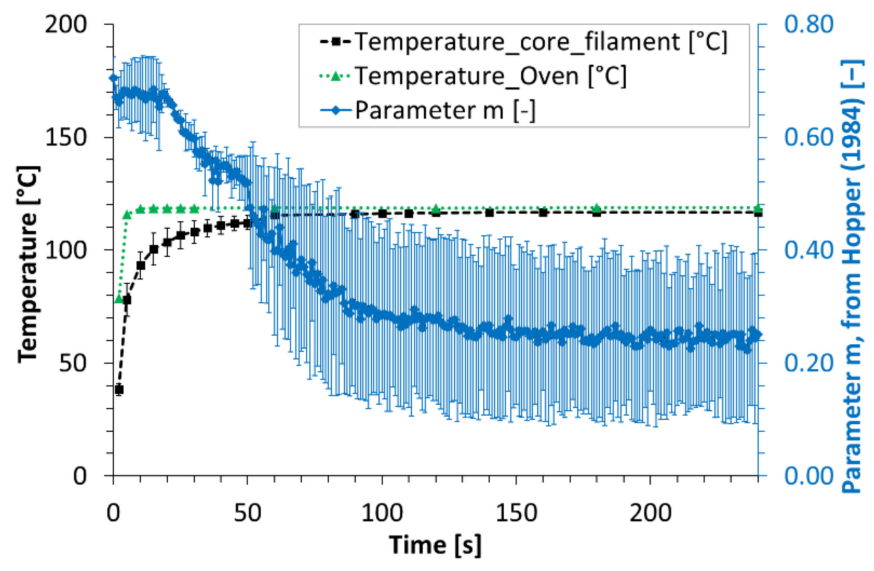

Figure 4. Theoretical evolution of the parameter B (from Sowinski and Jasion [19]) with parameter $\mathrm{m}$ (from Hopper [4]). Presentation of the acquired images of the thermoplastic filaments during a sintering trial at $120^{\circ} \mathrm{C}$ with the superimposition of their edge pixels (-), the successive fitted lemniscates (-) and their corresponding values of parameter $m$ (Hopper [4]), $B$ and $L_{\max }$ (normalized here as the ratio: $\mathrm{L}_{\max } / \mathrm{L}_{\max \_ \text {initial, }}$, and used to compute the parameter a (Sowinski and Jasion [19]), assessed by image analysis (a). Evolution of the parameter $B$ and $L_{\max }$ (normalized as $L_{\max } / L_{\text {max_initial }}$ ), assessed by image analysis during thermoplastic filament sintering at $120^{\circ} \mathrm{C}(\mathbf{b})$. Evolutions: (i) of temperatures measured in the oven environment; (ii) inside an extruded filament for $\mathrm{T}_{\text {set }}=120^{\circ} \mathrm{C}$; and (iii) of the values of the parameter $\mathrm{m}$ (Hopper [4]), assessed from parameter B (Sowinski and Jasion [19]) (c). 


\subsection{Assessment of the Material's Characteristic Viscous Sintering Time}

To determine the characteristic viscous sintering time, $t_{v s}$, the use of Hopper's abacus is based on the evolution of $\mathrm{m}^{2}$ (from $\mathrm{m}$ of Equation (4)) [4]. It decreases in a symmetric way, like the one of parameter $\mathrm{B}$, and allows the determination of the slope in the pure initial sintering step, from about $20 \mathrm{~s}$ to about $1 \mathrm{~min}$ at $120^{\circ} \mathrm{C}$ (Figure 5a). This experimental slope, $\left(d m^{2} / d t\right)_{\text {experimental, }}$ is assessed at $-6.0 \times 10^{-3} \pm 2.4 \times 10^{-3} \mathrm{~s}^{-1}$. Furthermore, the use of Hopper's abacus [4] makes it possible to draw a theoretical master curve (Figure 5b) and its polynomial fitting as: $\left(d m^{2} / d\left(t / t_{v s}\right)\right)_{\text {theoretical }}=f\left(m^{2}\right)$.

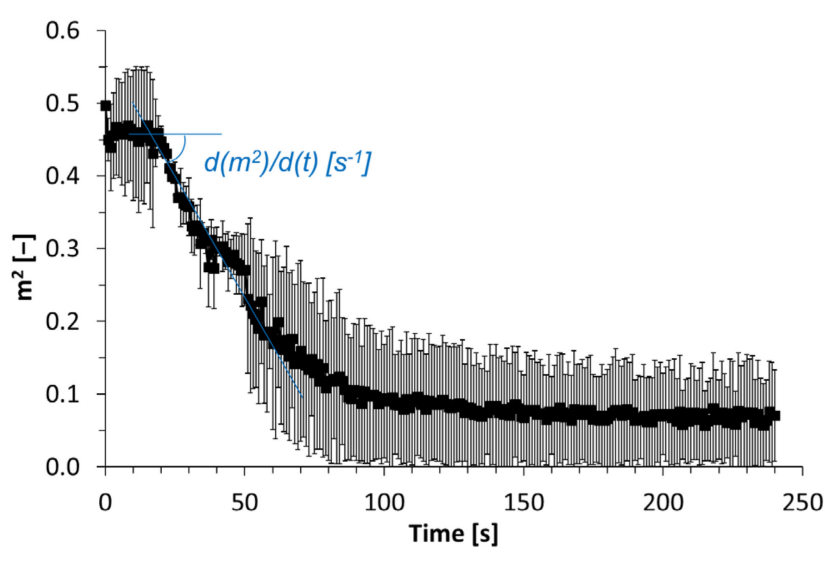

(a)

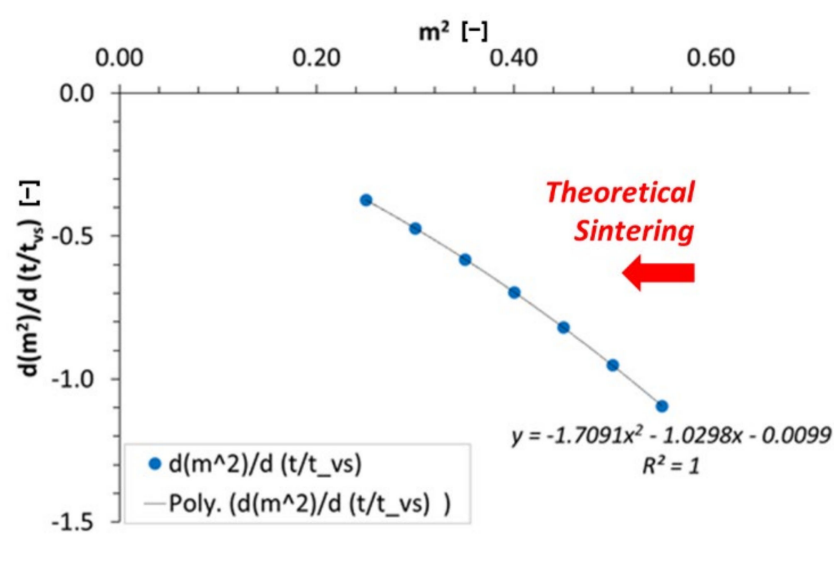

(b)

Figure 5. Evolution of the parameter $\mathrm{m}^{2}$ during the coalescence of thermoplastic filaments at $120{ }^{\circ} \mathrm{C}(\mathbf{a})$. Theoretical evolution of the decrease rate of $\mathrm{m}^{2}$ in relation to the characteristic viscous sintering time, $t_{v s}$, and plotted vs. $\mathrm{m}^{2}$ during theoretical sintering (master curve derived from Hopper's work; Hopper [4]) (b).

For typical values of $\mathrm{m}^{2}$ that decrease from 0.4 to 0.2 in the linear sintering domain from 20 to $60 \mathrm{~s}$, this makes it possible to determine the theoretical coefficient $\left(d m^{2} / d\left(t / t_{v s}\right)\right)_{\text {theoretical }}$ at -0.4727 , extrapolated for $\mathrm{m}^{2}=0.3$.

This value is linked to the experimental slope by the characteristic viscous sintering time, $t_{v s}$, as follows:

$$
\left(\frac{d m^{2}}{d\left(\frac{t}{t_{v s}}\right)}\right)_{\text {theoretical }}=t_{v s} \times\left(\frac{d m^{2}}{d t}\right)_{\text {experimental }},
$$

Such result leads to the determination of the characteristic sintering time: $t_{v s}=-0.4727 /-0.0060=78.8 \pm 31.8 \mathrm{~s}$. This value of $t_{v s}$ may be compared to the one previously assessed at about $80 \mathrm{~s}$ by the determination of the bonding neck length between sintered filaments and its associated growth rate [12]. They are similar and this result confirms the efficiency of the novel image analysis method leading to simulate the geometry of thermoplastic parts during their sintering. It also confirms the processing window previously defined for this biopolymer-based thermoplastic material, i.e., the plasticized zein, with the complete assessment of the sintering sequence, now possible, as the progressive decrease of the residual concavity, followed by swelling and spreading for a longer time. The evolution of the estimated parameters required to use Hopper's model are similar to those that allowed the determination of the bonding neck length alone, but in the present work, it corresponds to the exact monitoring of the filament geometry, progressively transformed into a homogeneous round melt obtained at high temperatures after the complete sintering of the filaments. 


\section{Discussion}

In this work, the ability of lemniscates of Booth to describe the contour of the crosssection of a pair of thermoplastic 3D printing filaments has been demonstrated in the model case of viscous sintering. The efficient analysis of images acquired during the viscous isothermal sintering of thermoplastic filaments allows the integral monitoring of the evolution of their overall edge shape. Indeed, such a side view shape follows their pure initial coalescence, up to about $60 \mathrm{~s}$, followed by viscoelastic swelling and spreading up to about $90 \mathrm{~s}$ with a radial straining of $112 \%$. The determination of the parametric equations of successive lemniscates of Booth that fit their evolving contour leads to the computation of the characteristic viscous sintering time of the considered material, checked at $t_{v s} \approx 80 \mathrm{~s}$, as previously found with a standard approach based on the determination of the bonding neck length between sintered parts. These results validate the enhanced images analysis approach proposed in the present work and its use for the assessment of sintering properties of a thermoplastic polymer in the molten state. In future works, the equations of lemniscates of Booth may be used to simulate the cross section's shape of juxtaposed filaments, as the local geometry of basic patterns constituting digital twins of 3D printed objects.

Supplementary Materials: The following are available online at https:/ /www.mdpi.com/article/10.3390/ polym13223965/s1, Script-Code ESM_1: Script (Matlab ${ }^{\circledR}$ ) to optimize the value of the shape parameter $\mathrm{B}$ of the polar equation of the fitted lemniscate. Video ESM_2: Sintering sequence at $120{ }^{\circ} \mathrm{C}$ and corresponding image analysis (1 frame/s acquired over $120 \mathrm{~s}$, accelerated 10 times and saved as a movie file). Plot of the successive shape of the filaments detected as edge pixels by image analysis ( -$)$ and the corresponding fitted lemniscates of Booth (-) in a polar coordinate system. Video ESM_3: Sintering sequence carried out at $120^{\circ} \mathrm{C}$ and its corresponding image analysis ( 1 frame/s acquired over $120 \mathrm{~s}$, accelerated 10 times and saved as a movie file). Images acquired during the sintering of two thermoplastic filaments at $120^{\circ} \mathrm{C}$ (8-bit coded, i.e., 256 grey levels) and superimposition of their edge ( -$)$ and the successive fitted lemniscates of Booth $(-)$.

Author Contributions: Conceptualization, Investigation, Writing—original draft preparation and writing—review and editing, L.C. and E.L.; Methodology and Writing—review and editing, A.-L.R. All authors have read and agreed to the published version of the manuscript.

Funding: This research received no external funding.

Institutional Review Board Statement: Not applicable.

Informed Consent Statement: Not applicable.

Data Availability Statement: Data are available as an accessible dataset cited in the manuscript as reference [20] (12 October 2020) https:/ / data.mendeley.com/datasets/gyd29mpt6b/1.

Acknowledgments: The authors would like to thank Denis Lourdin and Guy Della Valle (INRAE, Nantes) for helpful discussions, as well as Kévin Cochet, Patrice Papineau (INRAE, Nantes) and Yannick Madec (Univ. Nantes) for experimental support.

Conflicts of Interest: The authors declare no conflict of interest.

\section{References}

1. Turner, B.N.; Strong, R.; Gold, S.A. A review of melt extrusion additive manufacturing processes: I. Process design and modelling. Rapid Prototyp. J. 2014, 20, 192-204. [CrossRef]

2. Turner, B.N.; Gold, S.A. A review of melt extrusion additive manufacturing processes: II. Materials, dimensional accuracy, and surface roughness. Rapid Prototyp. J. 2015, 21, 250-261. [CrossRef]

3. Serdeczny, M.P.; Comminal, R.; Pedersen, D.B.; Spangenberg, J. Numerical simulations of the mesostructure formation in material extrusion additive manufacturing. Addit. Manuf. 2019, 28, 419-429. [CrossRef]

4. Hopper, R.W. Coalescence of Two Equal Cylinders: Results for Creeping Viscous Plane Flow Driven by Capillarity. J. Am. Cer. Soc. 1984, 67, C262-C264, Erratum in: 1985, 68, C138. [CrossRef]

5. Mcllroy, C.; Olmsted, P.D. Disentanglement effects on welding behaviour of polymer melts during the fused-filament-fabrication method for additive manufacturing. Polymer 2017, 123, 376-391. [CrossRef] 
6. Xin, L.; Boutaous, M.; Xin, S.H.; Siginer, D.A. Multiphysical modeling of the heating phase in the polymer powder bed fusion process. Addit. Manuf. 2017, 18, 121-135. [CrossRef]

7. Polychronopoulos, N.D.; Vlachopoulos, J. The role of heating and cooling in viscous sintering of pairs of spheres and pairs of cylinders. Rapid Prototyp. J. 2020, 26, 719-726. [CrossRef]

8. Bellehumeur, C.; Li, L.; Sun, Q.; Gu, P. Modeling of bond formation between polymer filaments in the fused deposition modeling process. J. Manuf. Process 2004, 6, 170-178. [CrossRef]

9. Boyd, J.; West, W.T.J.; Wu, S.; Takacs, E.S.; Vlachopoulos, J.; Thompson, M.R. Automated image analysis to characterize the melt densification stage of polymer sintering processes. Polym. Test. 2017, 60, 242-249. [CrossRef]

10. Bakrani Balani, S.; Chabert, F.; Nassiet, V.; Cantarel, A.; Garnier, C. Toward improvement of the properties of parts manufactured by FFF (Fused Filament Fabrication) through understanding the influence of temperature and rheological behaviour on the coalescence phenomenon. AIP Conf. Proc. 2017, 1896, 040008. [CrossRef]

11. Bakrani Balani, S.; Cantarel, A.; Chabert, F.; Nassiet, V. Influence of parameters controlling the extrusion step in fused filament fabrication (FFF) process applied to polymers using numerical simulation. AIP Conf. Proc. 2018, 1960, 140003. [CrossRef]

12. Chaunier, L.; Della Valle, G.; Lourdin, D.; Réguerre, A.-L.; Cochet, K.; Leroy, E. Viscous sintering kinetics of biopolymer filaments extruded for 3D printing. Polym. Test. 2019, 77, 105873. [CrossRef]

13. Chaunier, L.; Guessasma, S.; Belhabib, S.; Della Valle, G.; Lourdin, D.; Leroy, E. Material extrusion of plant biopolymers: Opportunities \& challenges for 3D printing. Addit. Manuf. 2018, 21, 220-233. [CrossRef]

14. Hopper, R.W. Coalescence of 2 viscous cylinders by capillarity. 1. Theory. J. Am. Cer. Soc. 1993, 76, 2947-2952. [CrossRef]

15. Hopper, R.W. Coalescence of 2 viscous cylinders by capillarity. 2. Shape evolution. J. Am. Cer. Soc. 1993, 76, 2953-2960. [CrossRef]

16. Frenkel, J. Viscous flow of crystalline bodies under the action of surface tension. J. Phys. (USSR) 1945, 9, 385-391.

17. Pokluda, O.; Bellehumeur, C.T.; Vlachopoulos, J. Modification of Frenkel's model for sintering. J. Am. Inst. Chem. Eng. 1997, 43, 3253-3256. [CrossRef]

18. Bordère, S.; Gendron, D.; Heintz, J.M.; Bernard, D. Monte Carlo prediction of non-Newtonian viscous sintering: Experimental validation for the two-glass-cylinder system. J. Am. Cer. Soc. 2005, 88, 2071-2078. [CrossRef]

19. Sowinski, K.; Jasion, P. Strength and stability of shells based on Booth lemniscate loaded with external pressure. Thin-Wall. Struct. 2019, 144, 106284. [CrossRef]

20. Chaunier, L.; Réguerre, A.L.; Leroy, E. Lemniscates fitting to the contour of thermoplastic filaments pairs during viscous sintering. Mendeley Data 2020. [CrossRef] 